\title{
Case Report \\ Diffuse Large B Cell Lymphoma Mimicking Granulomatosis with Polyangiitis
}

\author{
Mohammad E. Naffaa, ${ }^{1,2}$ Alexander P. Rozin,, ${ }^{1,2}$ Netanel Horowitz, ${ }^{2,3}$ Ofer Ben-Itzhak, \\ Yolanda Braun-Moscovici, ${ }^{1,2}$ and Alexandra Balbir-Gurman ${ }^{1,2}$ \\ ${ }^{1}$ The B. Shine Rheumatology Unit, Rambam Health Care Campus, 31096 Haifa, Israel \\ ${ }^{2}$ Rappaport Faculty of Medicine, Technion-Israel Institute of Technology, 31096 Haifa, Israel \\ ${ }^{3}$ Hematology Institute, Rambam Health Care Campus, 31096 Haifa, Israel \\ ${ }^{4}$ Pathology Institute, Rambam Health Care Campus, 31096 Haifa, Israel
}

Correspondence should be addressed to Mohammad E. Naffaa; m_naffaa@rambam.health.gov.il

Received 28 February 2016; Accepted 24 April 2016

Academic Editor: Gregory J. Tsay

Copyright (C) 2016 Mohammad E. Naffaa et al. This is an open access article distributed under the Creative Commons Attribution License, which permits unrestricted use, distribution, and reproduction in any medium, provided the original work is properly cited.

In a patient with systemic multiorgan disease with overlapping features, the differential diagnosis included infectious diseases, malignancies, and systemic autoimmune or inflammatory diseases. We present an unusual case of a young male with B cell lymphoma who presented with symptoms mimicking systemic vasculitis and review the existing literature.

\section{Introduction}

Granulomatosis with polyangiitis (GPA) is a small and medium-sized vessels vasculitis which is highly associated with positive anti-neutrophil cytoplasmic antibodies (ANCA), mainly with antibodies against proteinase 3 (PR3) [1]. Among reported clinical manifestations of GPA, necrotizing granulomatous inflammation in the upper respiratory tract, pulmonary nodules (often with cavitations), and necrotizing glomerulonephritis are prominent; their combination is a classic GPA triad [1]. Involvement of other organs and systems, such as the skin, eyes, ears, and peripheral nervous system, is less specific but definitely is not rare. The involvement of gastrointestinal tract (GIT) in GPA is infrequent [2].

\section{Case Presentation}

A 22-year-old male was admitted because of fever $\left(39.5^{\circ} \mathrm{C}\right)$, weakness, excessive sweating, vomiting, and weight loss during the previous month. His medical history was unremarkable, and physical examination did not show any pathological signs. Laboratory data revealed white blood count 2100 (WBC, 4000-10800/ $\mu \mathrm{L}$ ), hemoglobin 12.5 (Hb, 13-17 g/dL), and platelet count 113000 (PLT, 130000-350000/ $\mu \mathrm{L}$ ); aspartate aminotransferase 65 (AST, 5-40 U/L), alanine aminotransferase 63 (ALT, 30-65 U/L), alkaline phosphatase 123 (AlkPh, 30-115 U/L), lactate dehydrogenase 997 (LDH, 60$225 \mathrm{U} / \mathrm{L})$, creatinine $0.73(0.4-1.3 \mathrm{mg} / \mathrm{dL})$, blood urea nitrogen 8 (BUN, 5-20 mg/dL), creatine kinase 20 (CK, 35$300 \mathrm{U} / \mathrm{L})$, albumin 3.9 (3.4-5 g/dL), and ferritin 469 (15$300 \mathrm{ng} / \mathrm{mL}$ ); erythrocyte sedimentation rate $24 \mathrm{~mm} / 1$ hour (ESR, $<20 \mathrm{~mm} / 1$ hour) and C-reactive protein $18 \mathrm{mg} / \mathrm{L}$ (CRP, $0-5 \mathrm{mg} / \mathrm{L})$. Coagulation profile and thyroid function tests were normal. Urine dip-stick was negative for protein or blood. Blood, urine, throat, and stool cultures were all sterile. Serology tests, including human immunodeficiency virus, hepatitis B and C virus, Cytomegalovirus, Epstein Barr virus (EBV), Rickettsia, Chlamydia, Toxoplasma, Brucella, and Q-fever, were negative. Anti-nuclear (ANA) and antidsDNA antibodies were positive; anti-cardiolipin, and $\mathrm{B}_{2}$ glycoprotein antibodies and cryoglobulins were negative; complements C3 and C4 levels were normal; protein electrophoresis and immunoelectrophoresis were within normal limits. Test for anti-neutrophil cytoplasmic antibodies (ANCA) was positive for myeloperoxidase (MPO) and negative for proteinase 3 (PR3). Computed tomography (CT) 


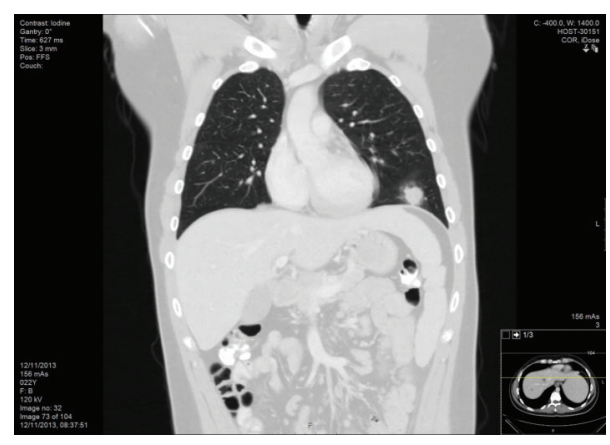

FIGURE 1: Coronal reconstruction of thoracic CT scan in lung window irregular pulmonary nodule with surrounding ground glass opacity in the left lower lobe of the lung.

demonstrated several bilateral peripheral nodular infiltrates in the lungs (Figure 1) and several nodular lesions in the liver and kidney; the spleen was mildly enlarged. Bone marrow biopsy was negative for granulomas and mycobacterium tuberculosis as well as for malignancy. The patient was discharged with a recommendation for ambulatory treatment with doxycycline for suspected atypical infection.

One month later, due to the ongoing fever, abdominal pain, repeated vomiting, constipation, weight loss, and loss of smelling ability, he was admitted again. Repeated CT scan showed resolution of previous pulmonary nodules with the concomitant appearance of several new ones. The number of hepatic nodules had increased with further enlargement of the spleen and the appearance of mild mesenteric lymphadenopathy. CT-guided fine needle aspiration of the pulmonary lesion failed and an open lung biopsy was performed. At that time, the patient was transferred to our hospital.

On admission the patient looked ill, pale, and weak. Heart and lung evaluation was unremarkable. There was mild tenderness of the upper abdomen without signs of peritoneal irritation or liver enlargement; the spleen was mildly enlarged and palpable. Repeated blood tests showed WBC $4200 / \mu \mathrm{L}$, HB $12.4 \mathrm{~g} / \mathrm{dL}$, PLT $248000 / \mu \mathrm{L}$, creatinine $0.65 \mathrm{mg} / \mathrm{dL}$, albumin $3.9 \mathrm{~g} / \mathrm{dL}$, AST $25 \mathrm{U} / \mathrm{L}$, ALT $43 \mathrm{U} / \mathrm{L}$, GGT $52 \mathrm{U} / \mathrm{L}$, ALKP $123 \mathrm{U} / \mathrm{L}, \mathrm{LDH} 234 \mathrm{U} / \mathrm{L}, \mathrm{CK} 20 \mathrm{U} / \mathrm{L}, \mathrm{CRP} 16.43 \mathrm{mg} / \mathrm{L}$, and ESR $24 \mathrm{~mm} / 1$ hour. Repeated ANA and anti-dsDNA were negative; the test for ANCA was pending. Gastroscopy showed only a small sliding hiatal hernia. Otolaryngologist assessment and brain magnetic resonance imaging were normal. Fundus evaluation did not show signs of retinal vasculitis. Transthoracic echocardiography was normal.

During the next several days the patient's condition deteriorated due to progressive weakness, abdominal pain, and repeated vomiting. After receiving the lung biopsy results which were summarized as necrotizing vasculitis and taking into account the existing findings from previous assessments (positive MPO, pulmonary nodules), the working diagnosis of ANCA-associated vasculitis was suggested and pulse therapy with methylprednisolone $1000 \mathrm{mg} /$ day for 3 consecutive days was introduced. After the first infusion the patient continued to complain of abdominal pain, constipation, and vomiting, and he refused to eat. Serial clinical abdominal assessments revealed epigastric tenderness without signs of peritoneal irritation. X-ray did not show free air in the abdominal cavity or signs of bowel obstruction. Abdominal ultrasonography demonstrated multiple hypoechoic hepatic lesions with mild ascites. Suddenly, the patient developed acute and severe epigastric pain, diffuse abdominal wall rigidity and rebound, marked tachycardia, and hypotension.

CT angiography of the abdomen demonstrated large amount of free air in the upper abdomen, peritoneal effusion, and a thickened small and large bowel wall with no signs of mesenteric arteries or veins thrombosis. The patient underwent emergent laparotomy which revealed multiple small necrotic areas in different segments of the small bowel; the involved part of small bowel was resected. The postoperative period was unremarkable. Pathologic examination of the resected small bowel showed high-grade EBV-associated diffuse large B cell lymphoma with signs of lymphomatoid granulomatosis and positive $\mathrm{IgH}$ rearrangement (Figure 2). The intestinal wall and the blood vessels showed extensive lymphoid infiltration. Later, repeated ANCA revealed negative results for MPO and PR3. The patient was treated with the CHOP-R protocol (cyclophosphamide, doxorubicin, vincristine, prednisone, and rituximab) with achievement of rapid clinical and hematological remission.

\section{Discussion}

We describe a unique case of a young male who developed high-grade EBV-associated diffuse large B cell lymphoma mimicking granulomatosis with polyangiitis (GPA). Our patient presented with constitutional symptoms (prolonged fever, severe fatigue, and weight loss), nasal involvement (anosmia), multiple pulmonary, hepatic, and spleen nodules, elevated inflammatory markers, anemia, transiently positive MPO, and findings on open lung biopsy suggestive of necrotizing vasculitis. All together, these findings were "strong" clues for the diagnosis of ANCA-associated vasculitis.

GPA is a small and medium-sized vessels vasculitis which is highly associated with positive ANCA, mainly with antibodies against PR3 [1]. Among reported clinical manifestations of GPA, necrotizing granulomatous inflammation in the upper respiratory tract, pulmonary nodules (often with cavitations), and necrotizing glomerulonephritis are prominent; their combination is a classic GPA triad [1]. Involvement of other organs and systems, such as the skin, eyes, ears, and peripheral nervous system, is less specific but definitely is not rare. The involvement of gastrointestinal tract (GIT) in GPA is infrequent [2]. Akbulut reported a patient with severe GPA who developed bowel perforation and sepsis and eventually died. The author reviewed thirteen similar cases and summarized the main clinical features of GIT involvement in GPA as follows: gingivitis, peptic ulcers, bloody diarrhea, bowel ulcerations, persistent perianal ulcers, pancreatitis, cholecystitis, small bowel perforations, and colitis [2]. Involvement of the GIT in GPA has been usually reported during relapses, in uncontrolled diseases, and late in the disease course (2-3 years later or more). The most common GIT complications in vasculitis in general and in GPA particularly are oral ulcers, intestinal ulcerations, bleeding, 


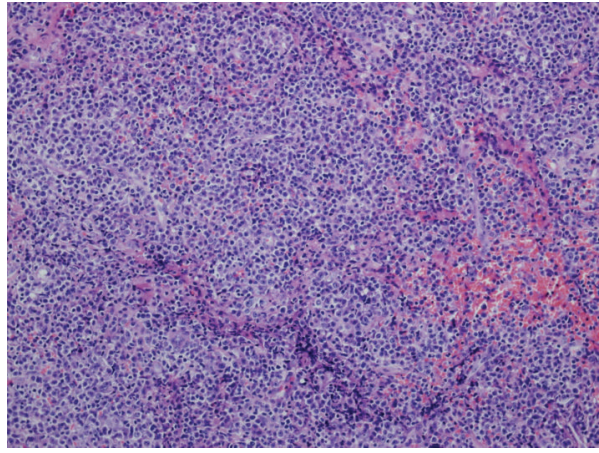

(a) Diffuse infiltrate of large lymphoid cells in the intestinal wall (Hematoxylin and Eosin, $\times 300$ )

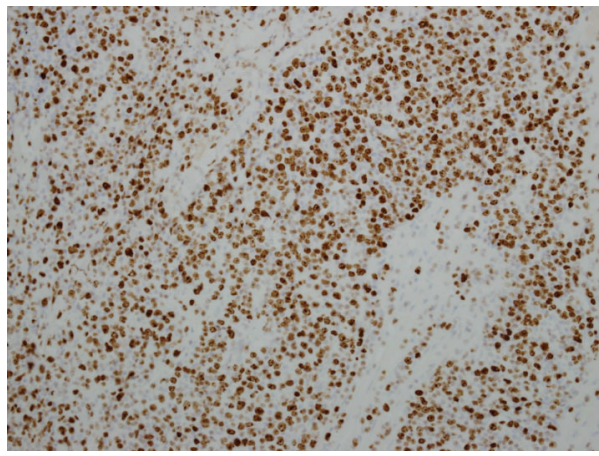

(c) Ki67 index over $90 \%$ of the large lymphoid cells (Immunostain for Ki67, ×200)

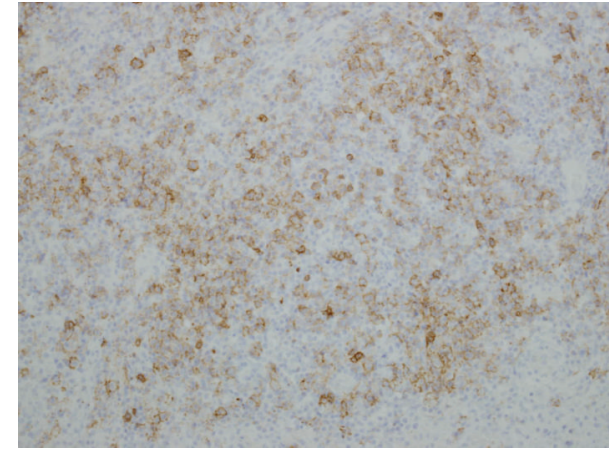

(b) Most of the large lymphoid cells show membranous staining for CD20 (Immunostain for CD20, $\times 300)$

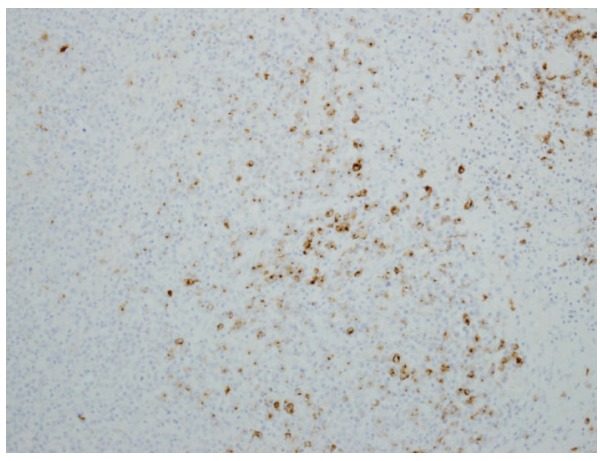

(d) Many of the large lymphoid cells show positive cytoplasmic staining for latent membrane protein of EBV (Immunostain for LMP-1, ×200)

FIGURE 2: Histopathologic examination of intestinal biopsy.

and perforations. In this regard, treatments such as corticosteroids and non-steroidal inflammatory drugs have been suggested as triggering agents. The presence of active vasculitisrelated changes at the sites of bowel perforations in about $61 \%$ of reported cases supports the idea that uncontrolled vasculitis in the intestinal wall was the main reason for perforation and not corticosteroids as was presumed previously [2]. Involvement of the GIT as a presenting symptom of GPA is extremely rare. We could find in the English language literature only 20 cases with GIT involvement as an early (less than one-year disease duration) or even initial GPA presentation (Table 1). Patients were mainly males $(15 / 20)$ with the mean age of 41.7 years (our patient was much younger) [3-22]. According to case descriptions, $4(20 \%)$ patients had appetite and weight loss, 9 (45\%) patients had oral and/or tongue ulcers, 6 (30\%) had diarrhea, $9(45 \%)$ had blood in stool, melena, or bloody diarrhea, $2(10 \%)$ had anal ulcers, and 1 had odynophagia (2\%); 9 (45\%) patients developed "acute abdomen" due to perforation, intra-abdominal bleeding, or obstruction. Our patient had abdominal symptoms for more than two months but never had oral ulcers, diarrhea, or GIT hemorrhage. Only in one reported patient was the GIT involvement an isolated GPA feature but was accompanied by fever [3] while, in the majority of cases, there were signs of multiorgan disease with almost an obligatory involvement of the upper airways (our patients had anosmia but not involvement of sinuses, eyes, or ears) and lungs (our patient had nodules without cavitations) and less often the kidneys (our patient did not have signs of nephritis).

Walton analyzed clinical and pathology features in 56 cases of GPA (Wegener's granulomatosis at that time). $\mathrm{He}$ found granulomatous lesions in the spleen (55.6\%), kidney (66.7), and liver (16.7\%) and fibrinoid necrosis in blood vessels in the spleen $(77.8 \%)$, kidney $(77.8 \%)$, liver (18.5\%), intestine (24.1\%), pancreas, and gallbladder (7.4\% each) [23]. One patient had severe refractory anosmia which later was complicated by nasal septum destruction and deafness (our patient also had anosmia but not structural or mucosal damage in the oral or nasal cavity). Described liver and spleen nodules were mainly microscopic findings but not macroscopic lesions on imaging films. Although the classic splenic manifestations of GPA are splenic infarcts, hemorrhage and spleen necrosis may also occur [24-27]. In one GPA case, multiple small bowel ulcers and perforations were accompanied by spleen infarctions [9]. Walton and Leggat described a patient with anosmia, lung involvement, multiorgan failure, and spleen granulomas [15].

Involvement of the liver is extremely rare in the course of GPA. Among liver manifestations, nonspecific nontraumatic hepatic hematoma and incomplete septal cirrhosis were 


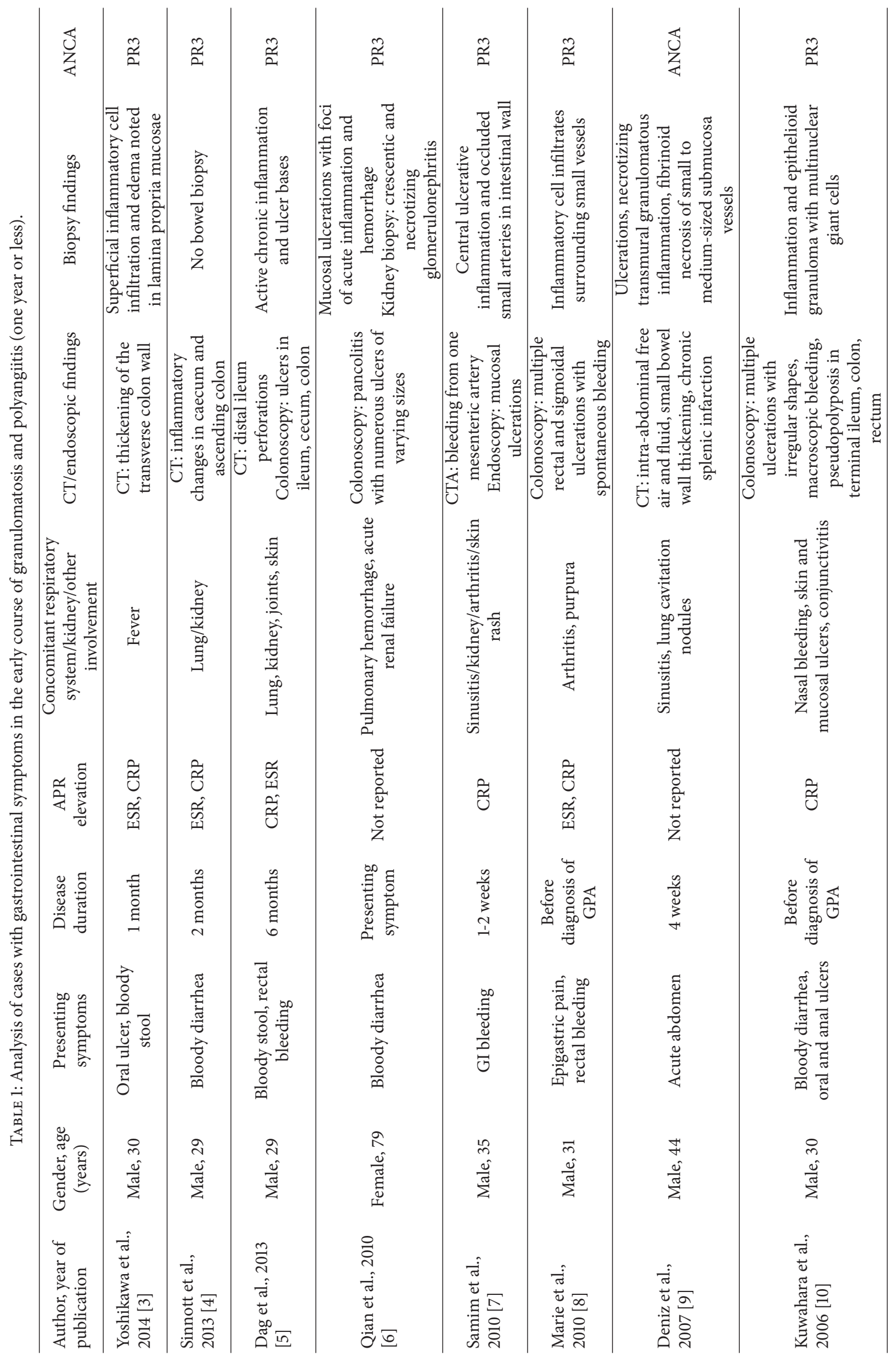




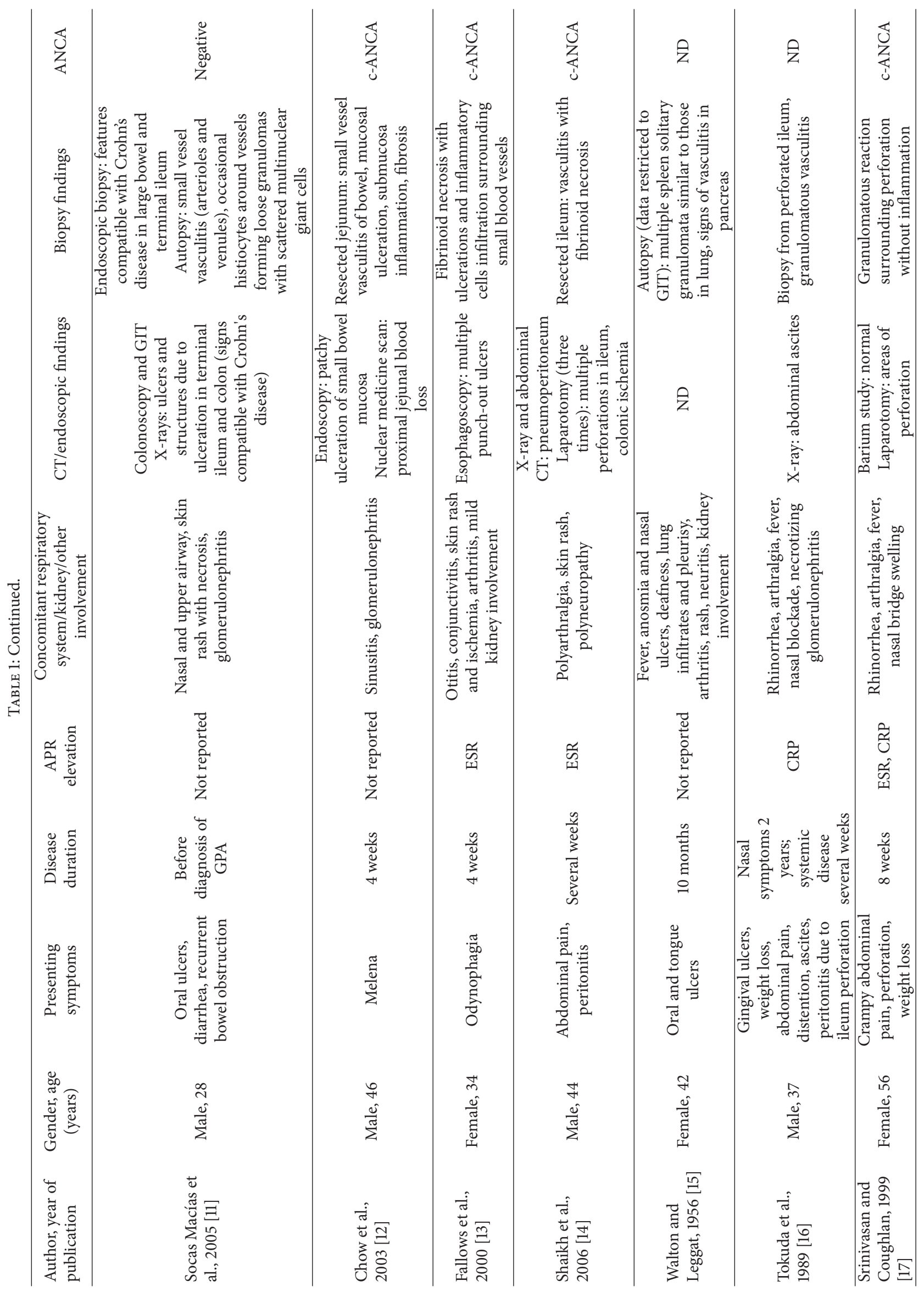




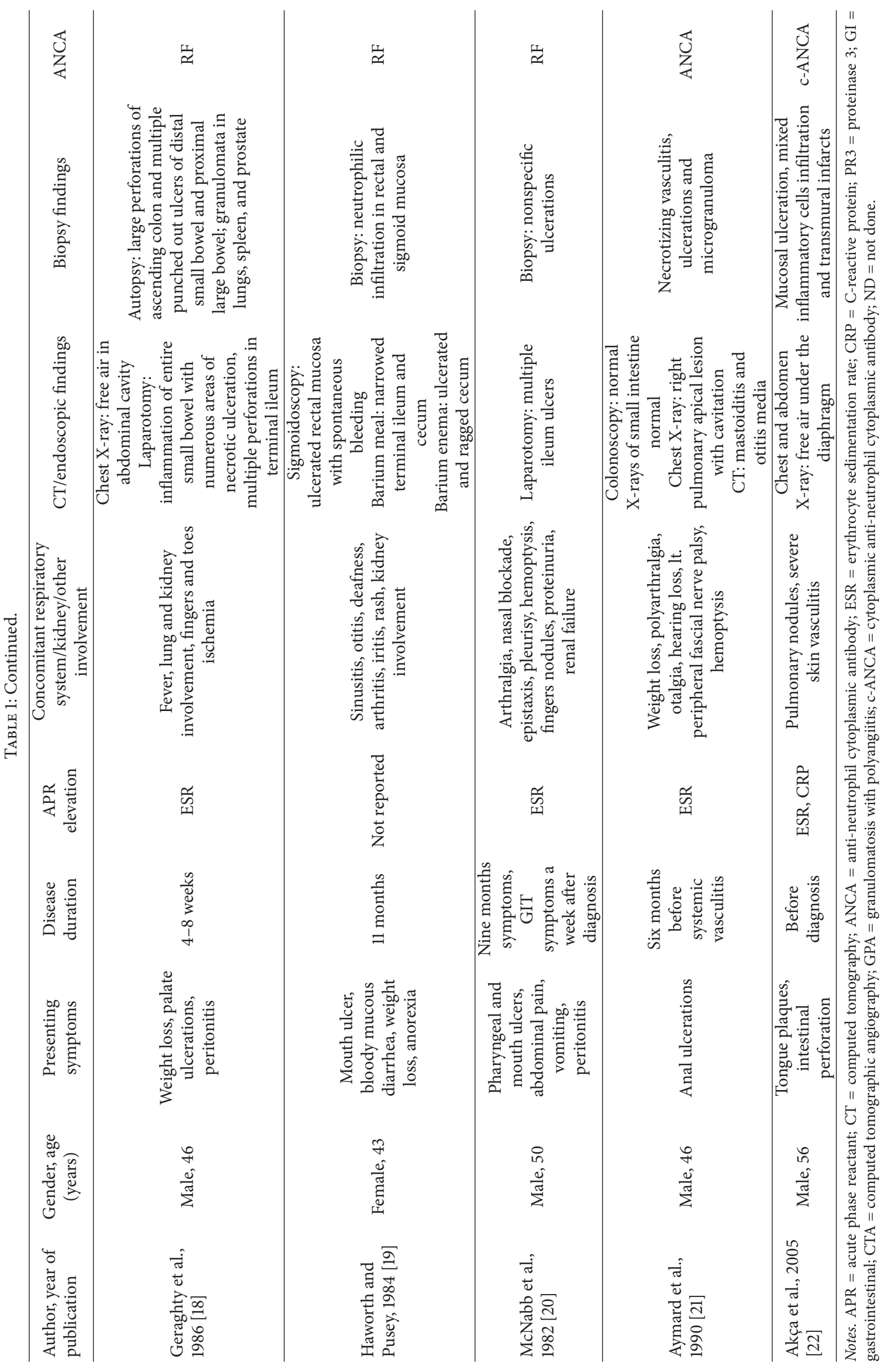


reported [28, 29]. Holl-Ulrich and Klass described a patient with necrotizing granulomatous changes in the liver, lungs, parotid glands, and skin in a GPA patient with fatal liver failure [30]. Our patient had multiple nodules in the liver, spleen, and kidneys. Liver biopsy in our patient demonstrated interface hepatitis without granulomas.

Before the 1990s, the main tools for assessment of GPA patients were X-rays with contrast and endoscopy. CT imaging became available later and was found to be very useful. It was obvious from analyzed cases that endoscopy had limited value in the diagnosis of vasculitis, as the main findings were mucosal ulcerations which are not specific for GPA (our patient did not have mucosal damage on endoscopy). In cases with active GIT bleeding on endoscopy, the diagnosis of vasculitis was more reasonable. Inability to perform the deep transmural biopsy during endoscopy also limited the diagnostic value of endoscopy; in the majority of reported cases GPA diagnosis was confirmed in resected bowel specimens or, unfortunately, in autopsies. In the 1980s, the main immunologic abnormality was positive rheumatoid factor (three cases); later, ANCA became a standard diagnostic tool and was reported positive in all but one case, c-ANCA or PR3 in particular (our patient had a transient MPO positive test).

The definitive diagnosis of GPA is based on tissue biopsy, mainly from upper respiratory tract or lung tissue. It is very clear from the Walton report that the presence of granulomas with neutrophils accumulation is a prominent and specific sign, while vascular wall fibrinoid necrosis is nonspecific and probably could only reflect the damage to the blood vessel wall by granuloma [23].

The diversity of GPA clinical manifestations that includes systemic signs (sort of "B-signs") and multiple organ and tissue involvement placed GPA in the list of great "imitators", as it shares various clinical features with other systemic diseases such as hematologic malignances and infections, making the definite diagnosis very challenging. Given the fact that our patient had a combination of listed features, positive ANCA, and findings on open lung biopsy suggestive of necrotizing vasculitis, the working diagnosis of GPA was justified, as was treatment with glucocorticoid pulse therapy. Our patient developed intestinal perforations. "Acute abdomen" with intestinal perforations has been described in GPA patients with GIT involvement $[9,16,17,22]$. In cases with acute abdomen, laparotomy is mandatory. In our patient, biopsies from resected bowel segments showed signs of diffuse large B cell lymphoma cells. Retrospective revision of lung pathology confirmed that the damage to the blood vessels was mainly due to an accumulation of lymphocytes in the involved tissue but not neutrophil accumulation or granulomas.

Different types of lymphoma may mimic different types of vasculitis. Lymphoma may be associated with ANCA positivity for both PR3 and MPO without vasculitis [31]. In a series of 119 patients with non-Hodgkin's lymphoma and 60 patients with Hodgkin's lymphoma, ANCA positivity was found in 8 patients ( 6 with p-ANCA and 2 with c-ANCA) with Hodgkin's lymphoma. None had vasculitis or rheumatic manifestations [32]. We could not explain the inconsistent results of positive MPO; in our institution the test for ANCA was negative. Despite the rare association between lymphoma and vasculitis, vasculitis in lymphoma patients mainly presents with skin purpuric rash and leukocytoclastic vasculitis on biopsy; our patient did not have any rash.

In searching the literature, we were able to find three cases of diffuse large B cell lymphoma mimicking GPA [33-35]. All these patients, like our patient, were males, aged between 20 and 40 years of age, and had pulmonary involvement; all had negative ANCA. None of these patients, in contrary to our patient, had liver or spleen involvement; one patient had renal involvement. In the case reported by Cohen et al., the patient was diagnosed with GPA and initially "responded" well to steroids and cyclophosphamide; only because of severe relapse under the treatment, revision of the biopsy leads to the proper diagnosis of lymphoma.

Did we have some clues to doubt the diagnosis of vasculitis in a patient who presented with systemic signs, multiorgan clinical presentations, presence of pulmonary nodules, positive MPO, and signs of vascular damage on open lung biopsy? A retrospective analysis of the case could teach us to pay attention to tiny discrepancies in the patient's condition: the anosmia in the absence of upper respiratory tract involvement was probably a "red herring"; the presence of multiple small and not consistent pulmonary nodules without cavitation is not a typical GPA feature; the presence of nodular lesions on CT imaging ("macroscopic") in the spleen, kidneys, and liver without signs of hemorrhage is not typical for GPA; severe dyspepsia (abdominal pain, vomiting, and constipation) without any mucosal damage (ulceration or bleeding) on upper endoscopy could not be explained by vasculitis, especially in the absence of diarrhea or intestinal/rectal bleeding. GPA is a disease with neutrophils activation and accumulation in organs and tissues; our patient did not have leukocytosis. Also, he had only mildly elevated ESR and CRP in contrast to very high levels in patients with GPA. It is unusual not to find c-ANCA (especially PR3) in patients with active GPA and multiorgan involvement as in our patient. Finally, the absence of granulomas in the involved lung tissue with only the pathological finding of vessel wall damage should raise the question regarding a diagnosis of vasculitis or a search for an alternative diagnosis.

We believe that in cases of atypical clinical course of systemic illness suggestive of vasculitis (GPA), the differential diagnosis should include lymphoma. Prompt efforts should be invested to confirm the diagnosis of vasculitis or to ensure that another condition, such as lymphoma, is not the cause of the disease. Our report pointed out the difficulties and obstacles on the way to making the correct diagnosis in patients with B cell lymphoma mimicking systemic GPA-like vasculitis.

\section{Competing Interests}

All authors declare no competing interests.

\section{Acknowledgments}

The authors thank Dr. Natalia Puchkov of the Department of Medical Imaging, Rambam Health Care Campus, who provided the CT images and their relevant legends. 


\section{References}

[1] P. M. K. Lutalo and D. P. D'Cruz, "Diagnosis and classification of granulomatosis with polyangiitis (aka Wegener's granulomatosis)," Journal of Autoimmunity, vol. 48-49, pp. 94-98, 2014.

[2] S. Akbulut, "Multiple ileal perforations in a patient with Wegener's granulomatosis: a case report and literature review," Journal of Gastrointestinal Surgery, vol. 16, no. 4, pp. 857-862, 2012.

[3] A. Yoshikawa, S. Yoshida, T. Takeuchi, Y. Fujiki, S. Makino, and T. Hanafusa, "Gastrointestinal involvement at the onset of granulomatosis with polyangiitis: a case report," Modern Rheumatology, 2015.

[4] J. D. Sinnott, P. Matthews, and S. Fletcher, "Colitis: an unusual presentation of wegener's granulomatosis," BMJ Case Reports, 2013.

[5] M. S. Dag, Y. Pehlivan, E. Tutar, and B. Kisacik, "Rituximab seems a promising therapeutic option in granulomatosis with polyangiitis with intestinal perforation: a case report and literature review," BMJ Case Reports, 2013.

[6] Q. Qian, L. Cornell, V. Chandan, R. Hartman, and S. Caples, "Hemorrhagic colitis as a presenting feature of Wegener granulomatosis," Journal of Gastrointestinal and Liver Diseases, vol. 19, no. 4, pp. 445-447, 2010.

[7] M. Samim, A. Pronk, and P. M. Verheijen, "Intestinal perforation as an early complication in Wegener's granulomatosis," World Journal of Gastrointestinal Surgery, vol. 2, no. 5, pp. 169171, 2010.

[8] I. Marie, L. Lahaxe, S. Josse, T. Lequerre, O. Vittecoq, and G. Savoye, "Rectal involvement revealing Wegener's disease," Scandinavian Journal of Rheumatology, vol. 39, no. 4, pp. 346-348, 2010.

[9] K. Deniz, H. S. Özşeker, Ş. Balas, E. Akpýnar, and C. Sökmensüer, "Intestinal involvement in Wegener's granulomatosis," Journal of Gastrointestinal and Liver Diseases, vol. 16, no. 3, pp. 329-331, 2007.

[10] Y. Kuwahara, Y. Shima, T. Tanaka et al., "Successful treatment with intravenous cyclophosphamide pulse therapy of severe intestinal involvement in Wegener's granulomatosis," Scandinavian Journal of Rheumatology, vol. 35, no. 3, pp. 243-245, 2006.

[11] M. Socas Macías, M. L. Sánchez Bernal, G. Suárez Artacho et al., "Severe intestinal involvement in Wegener's granulomatosis with negative c-ANCAs," Revista Espanola de Enfermedades Digestivas, vol. 97, no. 9, pp. 670-671, 2005.

[12] F. Y. F. Chow, D. Hooke, and P. G. Kerr, "Severe intestinal involvement in wegener's granulomatosis," Journal of Gastroenterology and Hepatology, vol. 18, no. 6, pp. 749-750, 2003.

[13] G. A. Fallows, S. F. Hamilton, D. S. Taylor, and S. B. Reddy, "Esophageal involvement in Wegener's granulomatosis: a case report and review of the literature," Canadian Journal of Gastroenterology, vol. 14, no. 5, pp. 449-451, 2000.

[14] F. M. Shaikh, C. B. Sabu, T. H. Peirce, and S. A. Naqvi, "Extensive intestinal ischaemic necrosis in Wegener's granulomatosis," Gut, vol. 55, no. 9, pp. 1368-1369, 2006.

[15] E. W. Walton and P. O. Leggat, "Wegener's granulomatosis," Journal of Clinical Pathology, vol. 9, no. 1, pp. 31-37, 1956.

[16] M. Tokuda, N. Kurata, H. Daikuhara et al., "Small intestinal perforation in Wegener's granulomatosis," Journal of Rheumatology, vol. 16, no. 4, pp. 547-549, 1989.

[17] U. Srinivasan and R. J. Coughlan, "Small intestinal perforation complicating Wegener's granulomatosis," Rheumatology, vol. 38, no. 3, pp. 289-290, 1999.
[18] J. Geraghty, I. R. Mackay, and D. C. Smith, "Intestinal perforation in Wegener's granulomatosis," Gut, vol. 27, no. 4, pp. 450451, 1986.

[19] S. J. Haworth and C. D. Pusey, "Severe intestinal involvement in Wegener's granulomatosis," Gut, vol. 25, no. 11, pp. 1296-1300, 1984.

[20] W. R. McNabb, M. S. Lennox, and J. A. Wedzicha, "Small intestinal perforation in Wegener's granulomatosis," Postgraduate Medical Journal, vol. 58, no. 676, pp. 123-125, 1982.

[21] B. Aymard, M. A. Bigard, H. Thompson, J. L. Schmutz, J. F. Finet, and J. Borrelly, "Perianal ulcer: an unusual presentation of Wegener's granulomatosis. Report of a case," Diseases of the Colon \& Rectum, vol. 33, no. 5, pp. 427-430, 1990.

[22] T. Akça, T. Çolak, M. Çglikulekçi, K. Öcal, and S. Aydin, "Intestinal perforation in Wegener's granulomatosis: a case report," Ulusal Travma ve Acil Cerrahi Dergisi, vol. 11, no. 4, pp. 348-351, 2005.

[23] E. W. Walton, "Giant-cell granuloma of the respiratory tract (Wegener's granulomatosis)," The British Medical Journal, vol. 2, article 265, 1958.

[24] J. C. Lee, I. Bringmann, and A. Aly, "Asymptomatic splenic infarct and retroperitoneal sepsis in patient with Wegener's granulomatosis," International Journal of Surgery Case Reports, vol. 3, no. 4, pp. 137-138, 2012.

[25] M. Martusewicz-Boros, I. Baranska, E. Wiatr, I. Bestry, and K. Roszkowski-Sliz, "Asymptomatic appearance of splenic infarction in Wegener's granulomatosis," Polish Journal of Radiology, vol. 76, no. 2, pp. 43-45, 2011.

[26] A. Ghinoi, N. Pipitone, A. Cavazza, L. Boiardi, and C. Salvarani, "Wegener granulomatosis with spleen infarction: case report and review of the literature," Seminars in Arthritis and Rheumatism, vol. 37, no. 5, pp. 328-333, 2008.

[27] D. Papaioannides, S. N. Nikas, M. Fotinou, and N. K. Akritidis, "Asymptomatic splenic infarction in Wegener's granulomatosis," Annals of the Rheumatic Diseases, vol. 61, no. 2, pp. 185-186, 2002.

[28] S. Doganay, E. Kocakoc, and M. Balaban, "Nontraumatic hepatic hematoma caused by Wegener's granulomatosis: an unusual cause of abdominal pain," New Zealand Medical Journal, vol. 123, no. 1318, pp. 73-78, 2010.

[29] Y. Zen, H. Sunagozaka, K. Tsuneyama et al., "Incomplete septal cirrhosis associated with Wegener's granulomatosis," Liver, vol. 22, no. 5, pp. 388-393, 2002.

[30] K. Holl-Ulrich and M. Klass, "Wegener's granulomatosis with granulomatous liver involvement," Clinical and Experimental Rheumatology, vol. 28, no. 1, supplement 57, pp. S88-S89, 2010.

[31] K. K. Zuckerman, L. Leventhal, and C. Wynne, "Positive cANCA in a patient with lymphoma and without vasculitis," Journal of Clinical Rheumatology, vol. 3, no. 5, pp. 279-281, 1997.

[32] T. Cil, A. Altintas, A. Isikdogan, and S. Batun, "Prevalence of antineutrophil cytoplasmic antibody positivity in patients with Hodgkin's and non-Hodgkin lymphoma: a single center experience," International Journal of Hematology, vol. 90, no. 1, pp. $52-57,2009$.

[33] Y. Cohen, G. Amir, G. Schibi, N. Amariglio, and A. Polliack, "Rapidly progressive diffuse large B-cell lymphoma with initial clinical presentation mimicking seronegative Wegener's granulomatosis," European Journal of Haematology, vol. 73, no. 2, pp. 134-138, 2004. 
[34] D. Gawryluk, J. Słodkowska, P. Dobkowski, and E. Wiatr, "Diffuse large B-cell lymphoma mimicking Wegener's granulomatosis," Pneumologia i Alergologia Polska, vol. 70, pp. 210-215, 2002.

[35] N. Miyahara, R. Eda, Y. Umemori et al., "Pulmonary lymphoma of large B-cell type mimicking Wegener's granulomatosis," Internal Medicine, vol. 40, no. 8, pp. 786-790, 2001. 


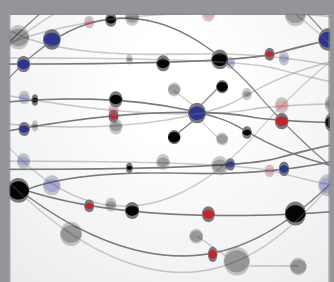

The Scientific World Journal
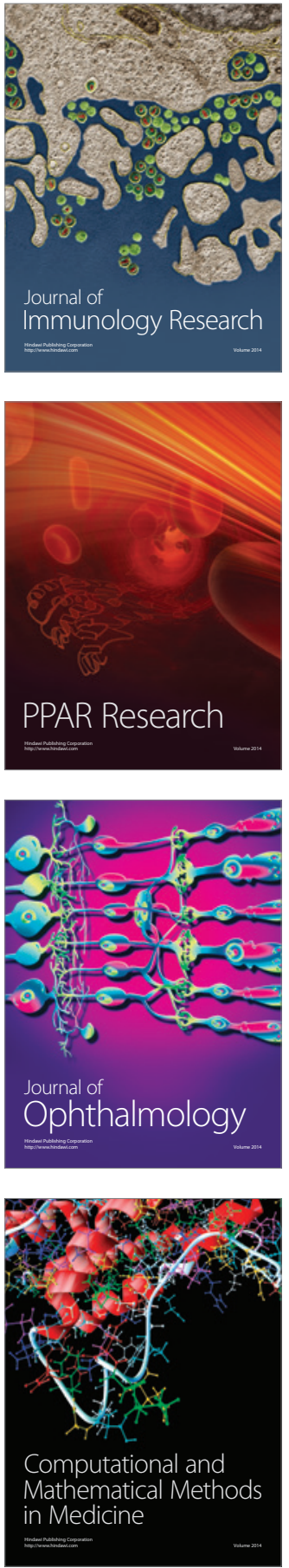

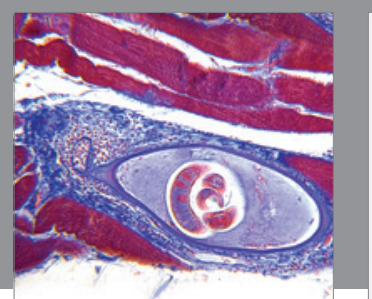

Gastroenterology Research and Practice

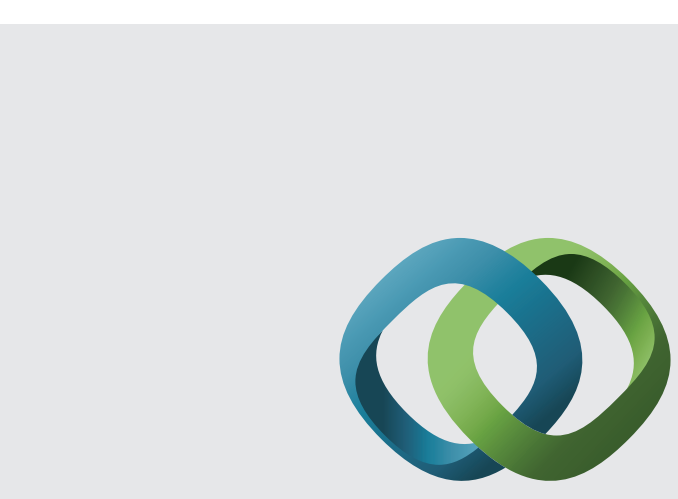

\section{Hindawi}

Submit your manuscripts at

http://www.hindawi.com
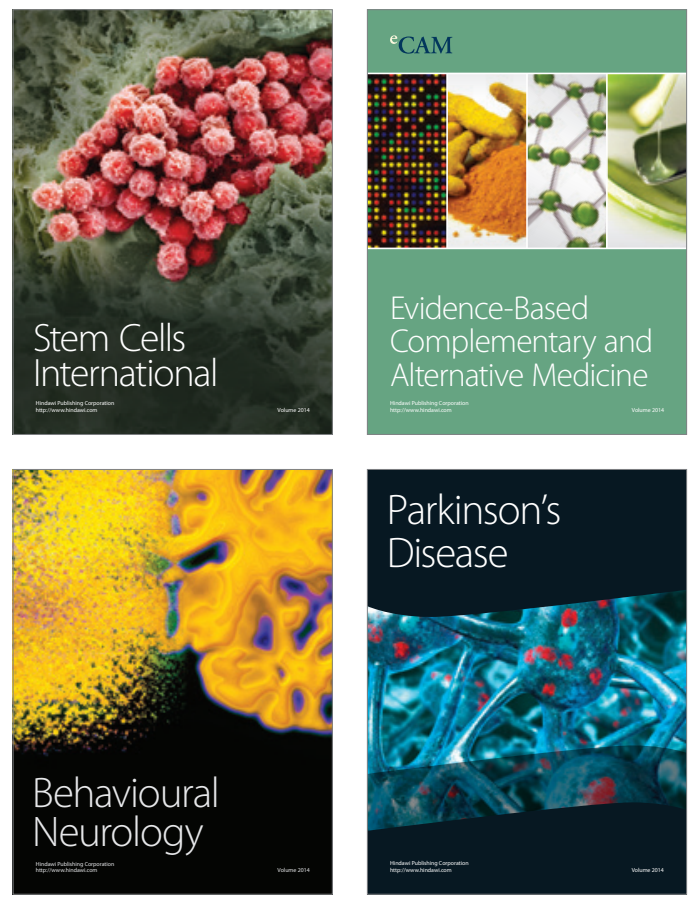
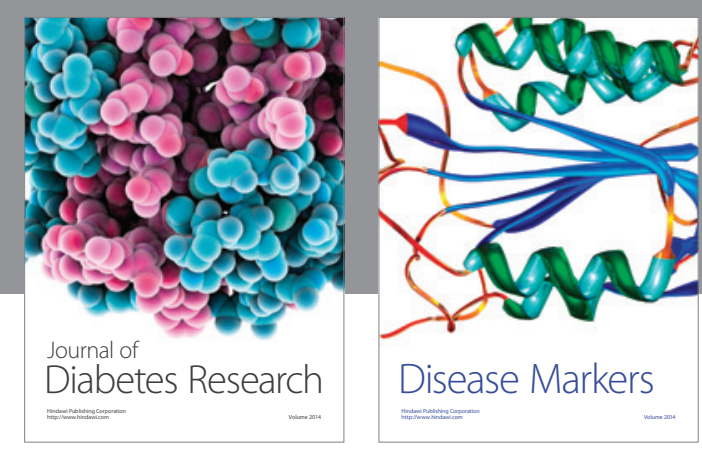

Disease Markers
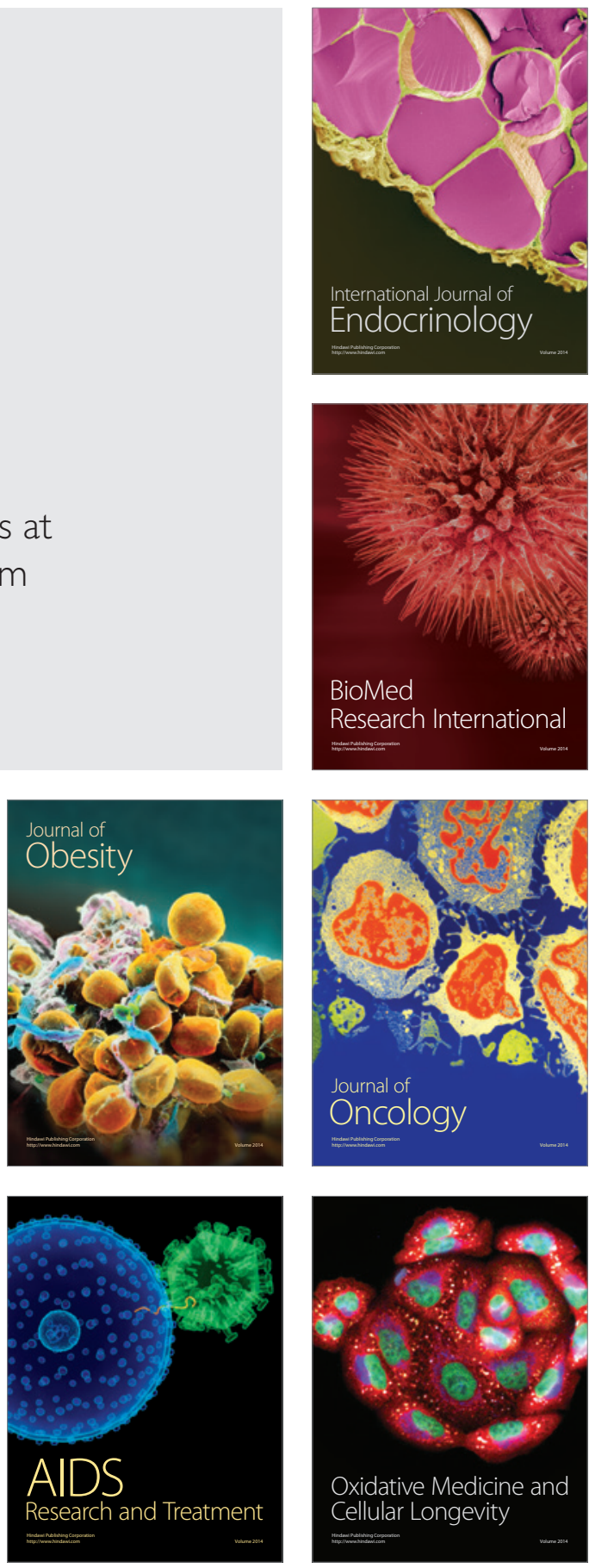